\title{
Magnetic Graphene-based Free-standing Composite Film for Electromagnetic Wave Absorption Applications Kaiyu Yang ${ }^{1, a}$, Xin Wang ${ }^{2, b^{*}}$ and Cunqing Ma $^{2, c}$
}

\author{
${ }^{1}$ State Key Laboratory of Automotive Simulation and Control, College of Automotive Engineering, \\ Jilin University, Changchun, 130022, P.R. China, \\ ${ }^{2}$ Key laboratory of Automobile Materials of MOE, College of Materials Science and Engineering, \\ Jilin University, Changchun, 130012, P.R. China \\ ayangky@jlu.edu.cn, ${ }^{\text {b* }}$ wang_xin@jlu.edu.cn, ${ }^{c} 1580786150 @ q q . c o m$
}

Keywords: Electromagnetic wave absorption, chemically modified graphene, composite, film.

\begin{abstract}
.
Carbon-based lightweight, thin, and self-standing composite film/paper could be a promising material for electromagnetic shielding applications because of their high flexibility, good electromagnetic properties, and high electrical conductivity. In this paper, a new-type composite of reduced graphene oxide (rGO) and $\mathrm{Fe}_{3} \mathrm{O}_{4}$ nanoparticles (F-NPs) has been investigated for potential applications in electromagnetic microwave absorbing film. The results indicated that with increasing the loading of F-NPs in the films, the values of saturation magnetization increased, contributing to an enhanced permeability of rGO/F-NPs composite films, while the dielectric loss decreased corresponding to a low conductivity. A maximum reflection loss of $-11.04 \mathrm{~dB}$ was obtained at 5.19 $\mathrm{GHz}$ for the composite with $100 \mathrm{mg}$ graphene oxide and $200 \mathrm{mg}$ F-NPs with a matching thickness of $3.0 \mathrm{~mm}$, and the effective absorption bandwidth with reflection loss values less than $-10 \mathrm{~dB}$ covered 2.8-8.6 GHz.
\end{abstract}

\section{Introduction}

The threat coming from electromagnetic pollution to human health and the surrounding environment, the potential commercial and military application have led to researching on electromagnetic (EM) interference shielding materials, especially on nanomaterials to meet all of the requirements such as lightweight, high-strength, wide and thin for the next generation of EM shielding application [1, 2]. In principle, there are three mechanisms of EM interference, namely reflection radiation, absorption of EM radiation, and scattering followed by absorption of EM waves, which determine desirable combined properties of high conductivity, dielectric and excellent magnetic characterization [2].

Graphene has attracted tremendous interest in both of the scientific and technological community since the successful isolation in 2004 due to its unique structure and excellent physicochemical and biological properties [3-5]. As one of its widely-known derivative, reduced graphene oxide (rGO) exhibits good dielectric properties and easily forming nanocomposites for potential applications in EM shielding and absorption either in form of nanostructured powders or films/papers [6-8]. rGO-based composites exhibited enhanced microwave absorption performance compared to the pure component. Chen et al. prepared rGO/epoxy composite sheets with various rGO loadings from 0.1 wt\% to $15 \mathrm{wt} \%$ in the epoxy solution and found that the reflection loss in X-band region (8.2-12.4 $\mathrm{GHz}$ ) showed maximum value of $-21 \mathrm{~dB}$ at the content of $15 \mathrm{wt} \%$ [6]. The high shielding efficiency was attributed to the high conductivity and large aspect ratio of single-layered rGO sheets. Magnetic graphene-based composites have also been studied extensively as a new type of absorbers. Zheng et al. studied the nucleation and growth $\mathrm{Fe}_{3} \mathrm{O}_{4}$ nanoparticles on $\mathrm{GO}$ nanosheet surface under different chemically environment and fabricated $\mathrm{rGO} / \mathrm{Fe}_{3} \mathrm{O}_{4}$ composites [9]. The value of reflection loss could be modulated by tuning the mass ratio of $\mathrm{Fe}_{3} \mathrm{O}_{4}$ and $\mathrm{GO}$, and a maximum value of $-8.75 \mathrm{~dB}$ at 8.11 
$\mathrm{GHz}$ with a thickness of $1.5 \mathrm{~mm}$ was obtained. By a combination of hydrothermal, annealing, and mixing processes, $\mathrm{rGO} / \mathrm{CoFe}$ nanocomposites have been prepared and the composite could be a promising lightweight and wide wavelength EM absorber because of its enhanced dielectric losses and magnetic losses [7]. Recently, $\mathrm{Li}$ et al synthesized nitrogen-doped graphene and $\mathrm{Fe}_{3} \mathrm{O}_{4}$ nanocrystal hybrids by solvothermal method. A maximum reflection loss value of $-65.3 \mathrm{~dB}$ was achieved at $6.8 \mathrm{GHz}$ with a matching thickness of $3.4 \mathrm{~mm}$ and the frequency of microwave absorption peak could be tuned [10]. The nanocrystal of $\mathrm{Fe}_{3} \mathrm{O}_{4}$ particles showed a superparamagnetic character and the nitrogen doping was considered to improve dielectric loss in the hybrid. By hydrothermal method, Zhu et al. synthesized rGO and $\mathrm{Fe}_{3} \mathrm{O}_{4}$ sub-microsphere composites and studied their dielectric properties and microwave absorbing performances [11]. The composites exhibited high chemical stability and low density with a maximum reflection loss of $-45 \mathrm{~dB}$ at $8.96 \mathrm{GHz}$ with the thickness of $3.5 \mathrm{~mm}$ and a better absorption bandwidth of $3.2 \mathrm{GHz}(<-10 \mathrm{~dB})$ compared to most magnetic materials and carbon-based composites.

In previous report, we fabricated $\mathrm{rGO} / \mathrm{Fe}_{3} \mathrm{O}_{4}$ nanocomposite film by a combination of solution-blending method and an improved step-by-step thermal annealing treatment [12]. Herein, we investigated the composition, crystalline structure, and microwave EM properties of the as-prepared films.

\section{Experimental}

In brief, colloidal solution of $\mathrm{GO} / \mathrm{Fe}_{3} \mathrm{O}_{4}$ nanoparticles (F-NPs) was prepared by a solution-blending method. To prepare rGO/F-NP composite film, the GO/F-NPs solution were heated step-by-step from room temperature to 60,120 , and $160{ }^{\circ} \mathrm{C}$ for $12 \mathrm{~h}$, respective [12].

The structure of the composite film was characterized by X-ray diffraction (XRD, D8tools; Bruker), and the component and composition were identified by X-ray photoelectron spectroscopy (XPS, Thermo ESCALAB 250 spectrometer). EM parameters were measured by a vector network analyzer (VNA, HP8720ES) in the range of 2-18 GHz.

\section{Results and Discussions}

Fig. 1 shows the XRD patterns of $\mathrm{rGO}, \mathrm{Fe}_{3} \mathrm{O}_{4}$ nanoparticles (F-NPs, F), and rGO with various F-NPs loadings (40, 120, $200 \mathrm{mg})$. For rGO/F composite films, at high amount of F-NPs, diffraction peaks at $30.2^{\circ}, 35.4^{\circ}, 43.0^{\circ}, 57.0^{\circ}$, and $62.5^{\circ}$ are appeared, which could be indexed as the characteristic (220), (311), (200), (511), and (440) reflections of the magnetite (file no. 19-0629). The as-prepared rGO film display a characteristic $2 \theta$ peak for the layered structure of graphene at about $22.6^{\circ}$, which can be ascribed to (002) reflection with a layer-to-layer distance, indicating a well-stacked structure of the films. Increasing loading of F-NPs, the characteristic peak is getting broader and smaller due to the addition of F-NPs between the layers, making a slightly larger $d$ spacing.

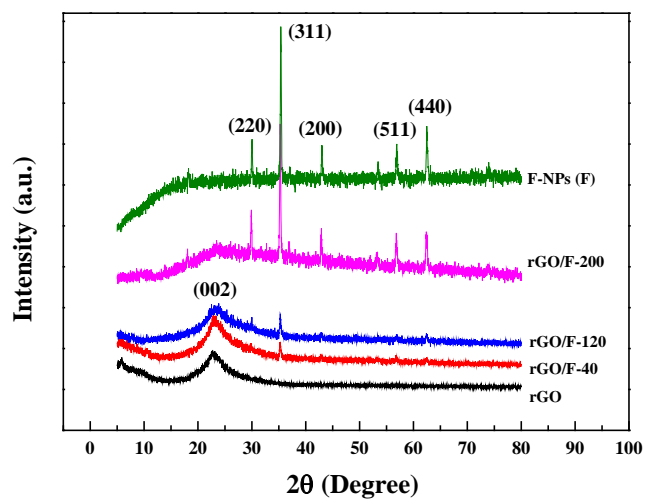

Fig.1 XRD patterns of samples F-NPs (F), rGO, rGO/F-40, rGO/F-120, and rGO/F-200 films. 

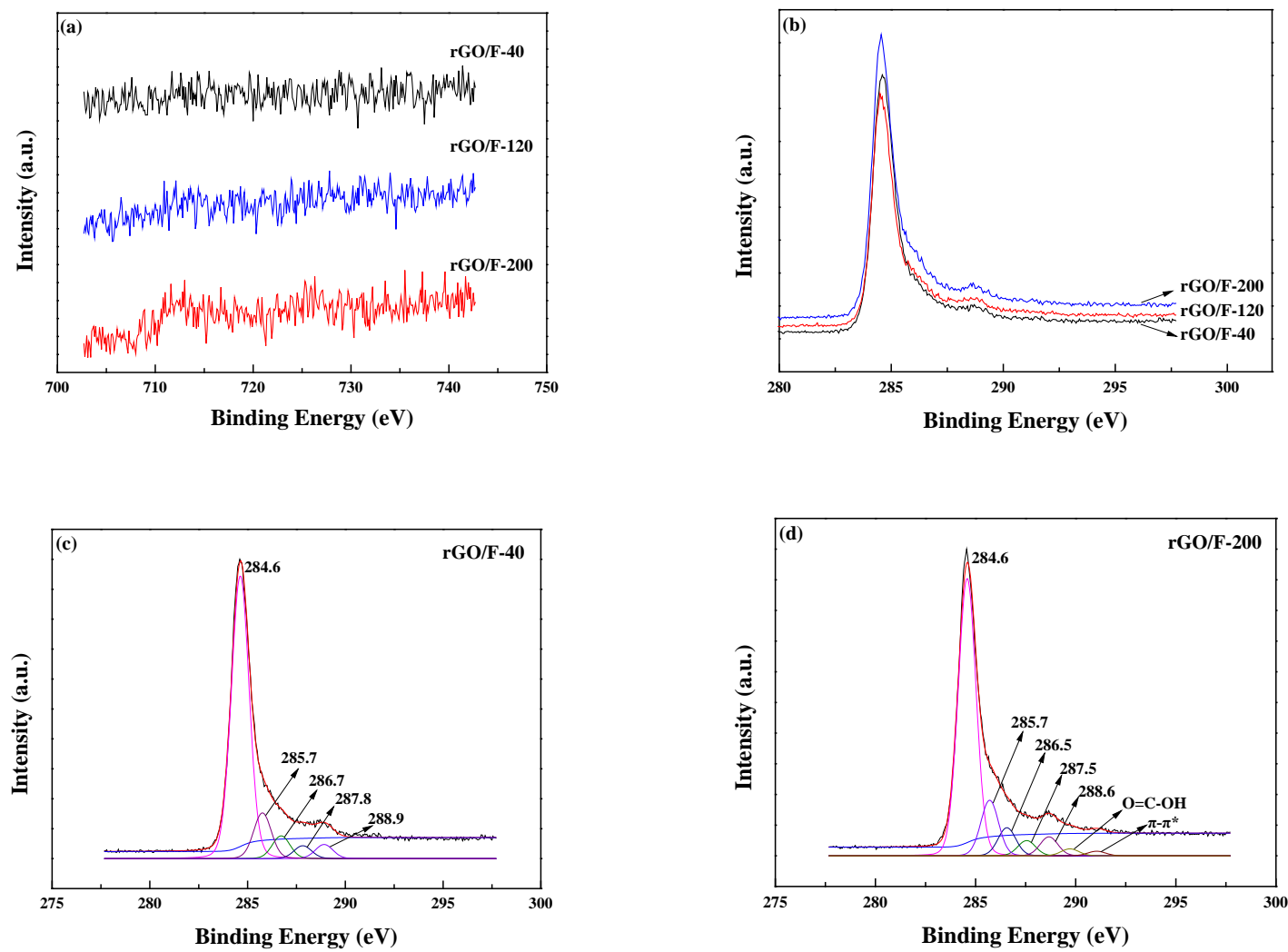

Fig.2 XPS spectra of Fe2p (a) and C1s (b) for samples rGO/F-40, rGO/F-120, and rGO/F-200; spectra of C1s after being fitted for samples rGO/F-40 (c) and rGO/F-200 (d).

According to SEM analysis, F-NPs in the composite dispersed uniformly on the surface of rGO film without occurring agglomeration [12]. To further identify the surface chemical composition and element states of the nanocomposite films, the XPS spectra were analyzed, as shown in Fig. 2. High resolution $\mathrm{Fe} 2 \mathrm{p}$ spectra of $\mathrm{rGO} / \mathrm{F}$ with various nanoparticle loadings were given in Fig. 2(a). It can be seen that very weak binding energy peaks at $710.6 \mathrm{eV}$ and $724.1 \mathrm{eV}$ appear, which belong to Fe 2p3/2 and Fe 2p 1/2, respectively, suggesting the smaller loading of F-NPs compared to the report in other literature [10]. Fig. 2(b-d) shows the high resolution of C 1s spectra of the nanocomposite films. The deconvoluted peaks with binding energies at 284.6, 285.7, 286.5, 287.5, and $288.6 \mathrm{eV}$ are attributed to the $\mathrm{sp}^{2} \mathrm{C}=\mathrm{C}, \mathrm{sp}^{3} \mathrm{C}-\mathrm{C}, \mathrm{C}-\mathrm{OH}, \mathrm{C}-\mathrm{O}-\mathrm{C}$, and $\mathrm{C}=\mathrm{O}$. At higher loading of $\mathrm{F}-\mathrm{NPs}, \mathrm{O}=\mathrm{C}-\mathrm{OH}$ and $\pi-\pi^{*}$ vibration satellite peaks appeared.
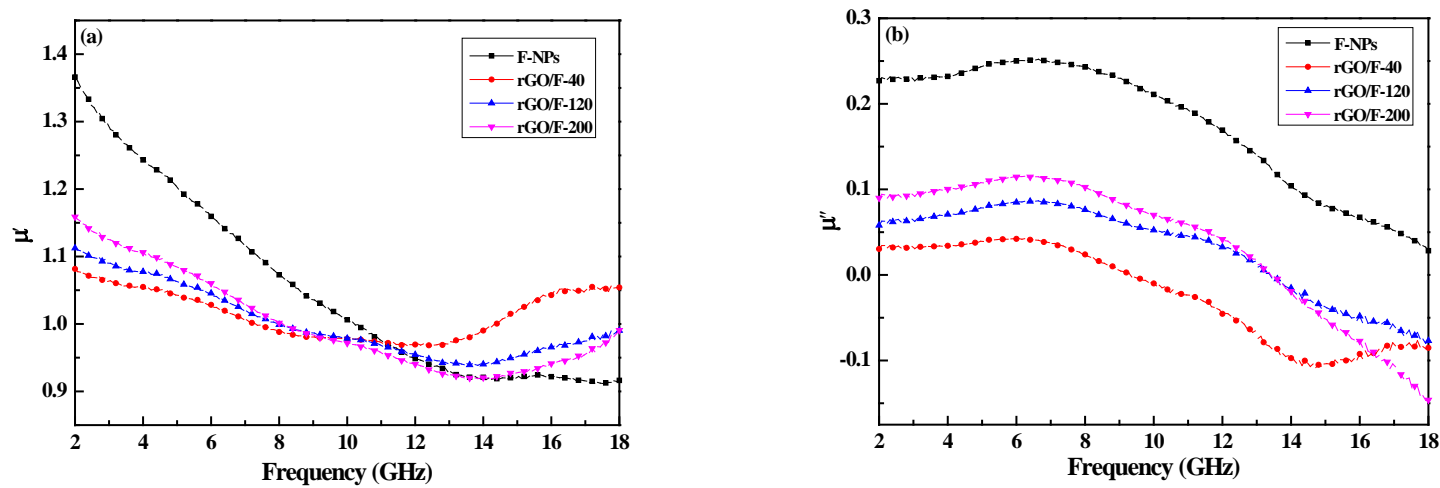

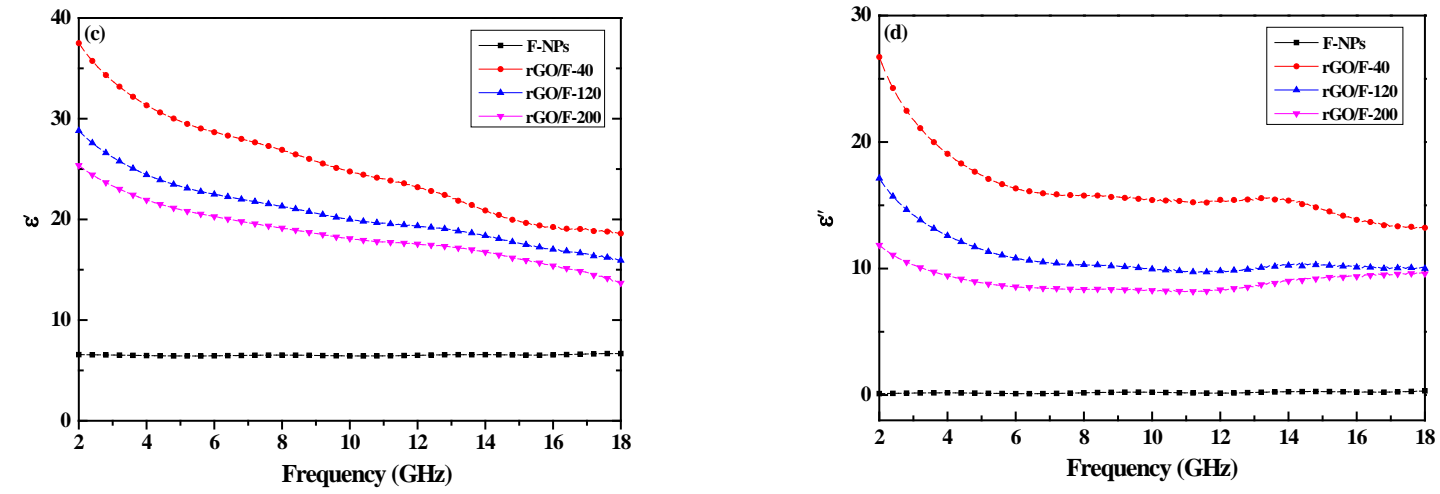

Fig.3 The spectrum characteristics of dielectric constant and permeability of samples F-NPs (a), rGO/F-40 (b), rGO/F-120 (c), and rGO/F-200 (d) films.

Fig. 3 shows the frequency-dependent permeability ( $\mu^{\prime}$, real part and $\mu^{\prime \prime}$, imaginary part) and permittivity $\left(\varepsilon^{\prime}\right.$, real part and $\varepsilon^{\prime \prime}$, imaginary part) curves for the pure F-NPs sample and rGO/F nanocomposite films. As shown in Fig. 3(a) and (b), nanocomposite film with higher F-NPs loading tends to has larger complex effective permeability $\left(\mu_{r}\right)$, i.e. higher storage capability and loss capability of magnetic energies, which is consistent with the measurement results of magnetic properties. In previous characterization, all of the nanocomposite films exhibited similar value of coercivity $\left(H_{c}\right)$, while the value of saturation magnetization $\left(M_{s}\right)$ for $\mathrm{rGO} / \mathrm{F}-200$ film was the largest.
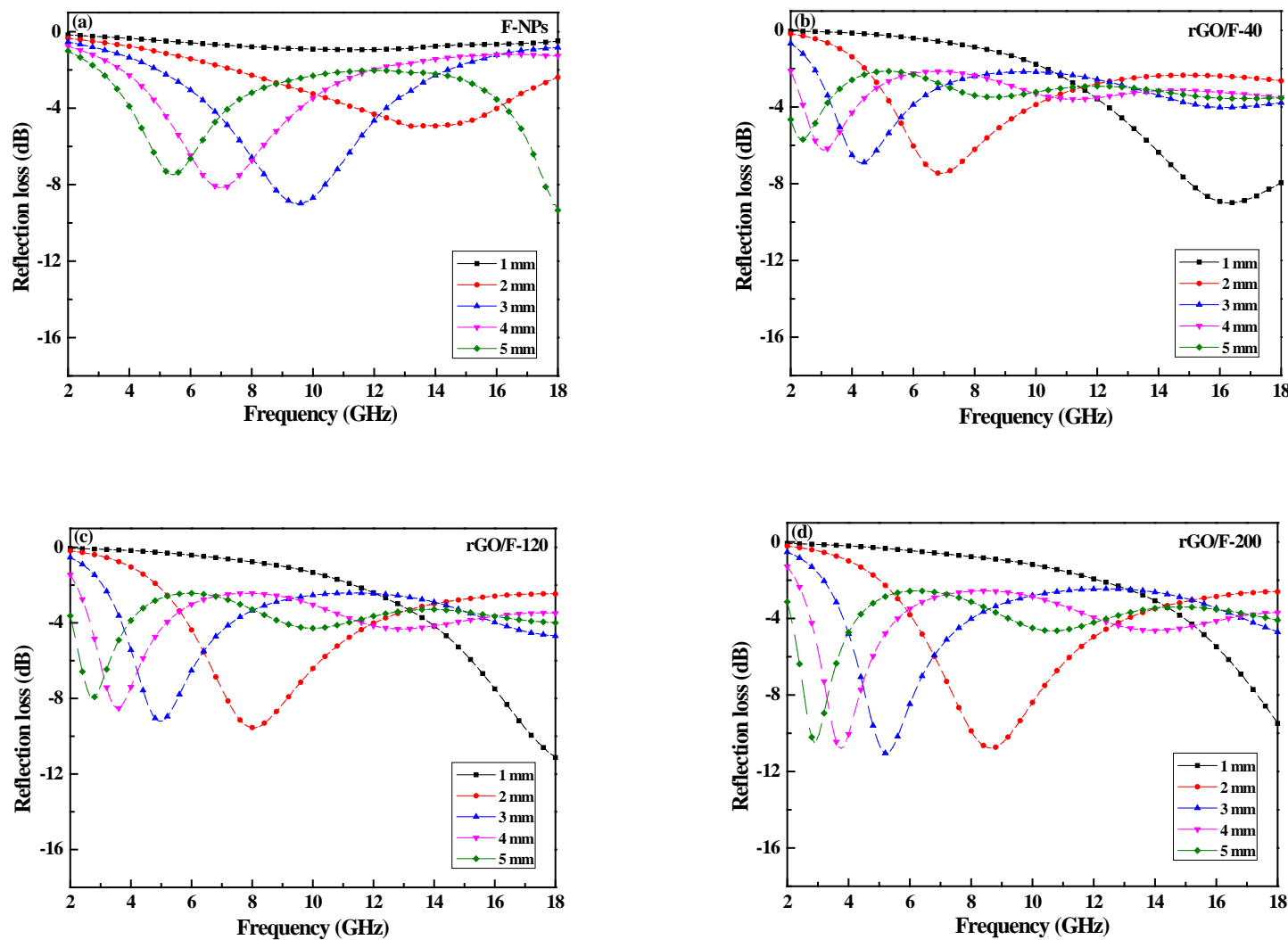

Fig.4 The reflection loss curves of samples F-NPs (a), rGO/F-40 (b), rGO/F-120 (c), and rGO/F-200 (d) films with frequency change.

Fig. 3(c) and (d) are the $\varepsilon^{\prime}$ and $\varepsilon^{\prime \prime}$ curves, showing a rapidly decrease with increasing the applied frequency for the nanocomposite samples, especially the small F-NPs loading at lower frequency. However, it should be mentioned that the nanocomposite films with $200 \mathrm{mg}$ F-NPs loading show a 
lowest complex effective permittivity $\left(\varepsilon_{r}\right)$ among the three composite samples, which can be ascribed to the decrease of conductivity of rGO film caused by the F-NPs.

Fig. 4 is the typical relationship between reflection loss and frequency of pure F-NPs, and the three nanocomposite films at different thickness. All of the values of reflection loss (RL) of pure F-NPs are lower than $-10 \mathrm{~dB}$ in the whole frequency range, while for the rGO/F-NPs samples, the microwave absorption performance is changed, tending to shift to low frequency. In addition, the absorption peak also shifts to a low frequency with the increase of film thickness. The film with $200 \mathrm{mg}$ F-NPs shows RL values exceeding $-10 \mathrm{~dB}$ in a frequency range of $2.8-8.6 \mathrm{GHz}$ and the maximum value can reach $-11.04 \mathrm{~dB}$ at a frequency of $5.19 \mathrm{GHz}$ with the absorber thickness of $3.0 \mathrm{~mm}$.

\section{Conclusion}

To control electromagnetic interference, rGO/F-NPs composite films have been fabricated by a facile and in-situ improved thermal reduction method as a novel type of microwave absorbers. Keeping the amount of rGO constant, the electromagnetic properties were found changing with the loading of F-NPs. The rGO/F-200 film exhibits effective absorption bandwidth (below $-10 \mathrm{~dB}$ ) of 2.8-8.6 GHz. The electromagnetic wave absorption performance could be turned by synergistic effect between F-NPs with magnetic loss and chemically modified graphene with dielectric loss.

\section{Acknowledgements}

This work was supported by the Natural Science Foundation of Jilin Province (Grant no. 20150101029JC).

\section{References}

[1] M.S. Sarto, A.G.D’Aloia, A. Tamburrano and G.De Bellis: IEEE Trans. Electromagn. Compat. Vol. 54 (2012), p. 17

[2] F. Shahzad, M. Alhabeb, C.B. Hatter, B. Anasori, S.M. Hong, C.M. Koo and Y. Gototsi: Science Vol. 353 (2016), p. 1137

[3] Z.Z. Wu, G.M. Zhou, L.C. Yin, W.C. Ren, F. Li and H.M. Cheng: Nano Energy Vol. 1 (2012), p. 107

[4] Z.Y. Wang and C.J. Liu: Nano Energy Vol. 11 (2015), p. 277

[5] C.N.R. Rao, in: Graphene and its Fascinating Attributes, edited by D.K. Pati Publications/World Scientific Publishing Co. Pte. Let. (2011)

[6] J.J. Liang, Y. Wang, Y. Huang, Y.F. Ma, Z.F. Liu, J.M. Cai, C.D. Zhang, H.J. Gao, Y.S. Chen: Carbon Vol. 47 (2009), p. 922

[7] Y. Ding, Q.L. Liao, S. Liu, H.J. Guo, Y.H. Sun, G.J. Zhang and Y. Zhang: Sci. Report Vol. 6 (2016), p. 1

[8] P. Bhattacharya and C.K. Das: J. Mater. Sci: Mater Electron Vol. 24 (2013), p. 1927

[9] J. Zheng, H.L. Lv, X.H. Lin, G.B. Ji, X.G. Li and Y.W. Du: J. Alloys Compd. Vol. 589 (2014), p.174

[10] Z.X. Li, X.H. Li, Y. Zong, G.G. Tan, Y. Sun, Y.Y. Lan, M. He, Z.Y. Ren and X.L. Zheng: Carbon Vol. 115 (2017), p.493

[11] L.Y. Zhu, X.J. Zeng, X.P. Li and R.H. Yu: J. Magn. Magn. Mater. Vol. 426 (2017), p.114

[12] C.Q. Ma, K.Y. Yang, Lili Wang and X. Wang: J. Appl. Biomater. Funct. Mater Vol. 15 (2017), p. S1 